\title{
Cardiovascular Responses to Submaximal Exercise Stress Test in Adolescents with High Visceral Fatness
}

\author{
Shweta Parikh', Sushil Kumar Singh ${ }^{2}$, Hasmukh Shah ${ }^{2, *}$
}

\section{Shweta Parikh' ${ }^{1}$, Sushil Kumar Singh², Hasmukh Shah ${ }^{2, *}$ \\ ${ }^{1} K M$ Patel Institute of Physiotherapy, Bhaikaka University, Karamsad, Gujarat, INDIA. \\ ${ }^{2}$ Department of Physiology, \\ Pramukhswami Medical College, Bhaikaka University, Karamsad, \\ Gujarat, INDIA.}

\section{*Correspondence}

\section{Dr. Hasmukh D. Shah,}

Professor \& Head,

Department of Physiology,

Pramukhswami Medical College \&

Bhaikaka Universtiy, Karamsad, Anand, Gujarat INDIA.

Phone: +919879731388

Email:drhasmukhshah0505@gmail. com

\section{History}

- Submission Date: 10-02-2021;

- Review completed: 12-03-2021.

- Accepted Date: 24-03-2021.

DOI : 10.5530/ijcep.2021.8.1.4

Article Available online

http://www.ijcep.org

\section{Copyright}

(C) 2021 Phcog.Net. This is an openaccess article distributed under the terms of the Creative Commons Attribution 4.0 International license.

\begin{abstract}
Background and Aim: Visceral adiposity per se was associated with risk of developing hypertension in future. There is escalating trend of visceral obesity in Indian adolescents. Nonetheless, the Asian Indian population is known to have an affinity for central fat deposition than peripheral fat. Meanwhile, the association of visceral adiposity and cardiovascular response to exercise in Indian adolescents of 18-19 years' age group has not been well described so far. Therefore, the present study was undertaken. Methods: A Cross-sectional study was conducted on 120 non-athletes' (60 males and 60 females) late Indian Adolescents of 18-19 years' age group. Visceral Fat (VF) and Other Body composition were measured by Bioelectrical Impedance technique using Omron HBF 302 body fat analyzer. The participants were customized into two groups based on their Visceral Fat Level (0-9: Normal VF group; > 9: High VF group). Exercise Testing was done by treadmill exercise test following standard Bruce protocol. Cardiovascular reactivity (Heart Rate, Systolic and Diastolic Blood Pressure) was checked at the end of each stage of Bruce Protocol. Results: Increased SBP and DBP responses to treadmill exercise tests at different stages were seen in high VF groups in both the genders. However, no abnormal reactivity was observed in high VF groups of both the genders. Conclusion: During treadmill exercise stress test, high blood pressure reactivity was seen in high visceral fat adolescents as compared to normal visceral fat adolescents, which was primarily due to their high resting blood pressure. Adolescents with normal visceral fat were competent to cross more number of Bruce stages than high visceral fat Indian adolescents. Visceral fat had a stronger influence on baseline cardiovascular parameters like systolic and diastolic blood pressure as well as blood pressure parameter during exercise than total body fat.
\end{abstract}

Key words: Visceral fat, Exercise Stress Test, Adolescents, Blood pressure, Heart rate.

\section{INTRODUCTION}

Cardiorespiratory or aerobic fitness was assessed by dynamic exercise test. Blood pressure (BP) reactivity to stress and chronotropic response are the best assessed by dynamic exercise test. ${ }^{[1]}$ Blood pressure reactivity to exercise stress test is different in all individuals. Normally rise in systolic blood pressure and heart rate are considered as normal physiological response. ${ }^{[2]}$ Exaggerated systolic blood pressure (SBP) after exercise and failure to rise after the exercise are considered as abnormal blood pressure response. Abnormal blood pressure response to exercise is one of the risk factors for cardiovascular health and it is responsible for the development of hypertension in normotensive individuals in future. ${ }^{[3]}$ American Heart Association and American College of Cardiology considered abnormal blood pressure response as warning sign for developing hypertension in normotensive. Blood pressure reactivity to exercise stress test unmasks the possibility of hypertension in future. ${ }^{[4]}$

Blood pressure reactivity to exercise which may be normal or abnormal is affected by multiple factors like genetic inheritance and ethnicity, age, gender,

body composition and physical activity level. ${ }^{[4,5]}$ Body fat percentage and body fat distribution are different even in individual with same body mass index (BMI). Visceral fatness affects the blood pressure. Increased BP at rest and heart rate are seen in individuals with high BMI, body fat mass (BFM) and with high visceral fat (VF). Blood pressure reactivity is also affected by tension of vascular wall, smoothness of vascular wall, elasticity of vascular wall and also depends on modulation of autonomic nervous system. ${ }^{[6]}$ Increased vagal tone is very well documented in athletes and physically active persons while increase sympathetic activity is seen in persons living with chronic environmental stress. Pre-existing metabolic and cardiovascular diseases are also affecting blood pressure reactivity. ${ }^{[6,7]}$

The period of adolescence is a preparatory period for adulthood. During adolescence, structural and hormonal changes were observed. Adolescents' health is big concern now-a-days mainly due to their life style changes. Sedentary lifestyle and abnormal eating behavior are key issues of adolescents. Sedentary life make them victim of centrally obese

Cite this article: Parikh S, Singh SK, Shah H. Cardiovascular Responses to Submaximal Exercise Stress Test in Adolescents with High Visceral Fatness. Int J Clin Exp Physiol. 2021;8(1):11-6. 
due to high visceral fatness. Hence during this period, assessment of blood pressure reactivity to exercise stress is a guiding force for making preventive strategy. Hypotensive or exaggerated blood pressure reactivity unmask possibility of hypertension in future. ${ }^{[8]}$

Treadmill exercise stress testing is one the best non-invasive method. Treadmill exercise testing is reliable and economic test which is easily repeatable. It is commonly used for diagnostic purpose and for determination of functional capacities in asymptomatic participants, as well prognostic purpose for routine follow-up in cardiac patients. ${ }^{[7,9]}$ Cardiorespiratory fitness can be easily assessed by Treadmill test in adolescents with low or minimum risk. Blood pressure and heart rate responses to the exercise can be assessed to unmask hypertension in future in normotensives. Recovery parameters also worked as a guiding tool in individual with different body mass and different fat distribution. Therefore, the present study was undertaken to assess the cardiovascular responses to submaximal treadmill exercise stress test in Indian adolescents of 18-19 years of age group.

\section{MATERIALS AND METHODS}

The present study was approved by Institutional Ethics Committee. Undergraduate students studying at H. M. Patel Centre for Medical Care and Education (Medical, Physiotherapy, Nursing, B.Sc. Medical Laboratory Technology) and the vicinity of Shree Krishna Hospital, Karamsad were recruited into the study after obtaining written informed consent. In this cross sectional study, 120 apparently healthy adolescents (60 males and 60 females) of 18-19 years age group were enrolled. Participants with history of cardiovascular diseases, diabetes, respiratory diseases or any other physical disability were excluded from the study. Participants with history of smoking and alcohol and other chronic diseases were also excluded from the study. Participants were instructed to avoid taking tea, coffee or any other stimulants and heavy meals before $3 \mathrm{hr}$ of exercise test and not to be engaged in any type of physical exercise at least $12 \mathrm{hr}$ prior to exercise test.

\section{Anthropometric Measurements}

Anthropometric parameters were measured with least clothing and bare feet. The height was measured using a meter scale without footwear to the nearest $0.1 \mathrm{~cm}$. Waist Circumference (WC) was measured at the midpoint between the lower costal margin and the highest point on the iliac crest to the nearest $0.5 \mathrm{~cm}$ at the end of normal expiration. Body weight was measured and BMI was calculated. Total Body Fat (TBF) percentage and VF were measured by bioelectrical impedance technique using Omron HBF-302 Body Fat Analyzer. Fat Mass (FM) was calculated based on total body fat percentage into body weight. Fat Free Mass (FFM) was calculated by subtracting fat mass from total body weight. The participants were customized into two groups based on their VF level (0-9: Normal VF group; > 9: High VF group).

\section{Exercise Testing by Treadmill Stress Test}

Cardiovascular parameters such as $\mathrm{HR}, \mathrm{SBP}$ and diastolic blood pressure (DBP) were measured by Omron T8 (HEM757A4-C1) before the exercise with all standard precautions. Cardiovascular or blood pressure response to exercise was tested by submaximal Treadmill Exercise Stress Test following the standard Bruce protocol as per proposed guidelines of the American College of Sports and Medicine and the American Heart Association. Bruce's treadmill exercise test is a continuous, incremental test which consists of 7 stages and each stage lasts for $3 \mathrm{~min}$. Exercise speed and grade were increased during each next stage as per the Bruce protocol. BP and HR were recorded at the end of each stage of exercise. Participants were asked to continue exercise until they reach $85 \%$ of their age adjusted predicted maximum HR or develop cardio-respiratory symptoms or Borg Scale of Perceived Exertion $\geq 15$.

The total duration of exercise was recorded in minutes and seconds. Recovery BP and HR were recorded after $2 \mathrm{~min}, 4 \mathrm{~min}$ and $6 \mathrm{~min}$ of cessation of exercise and they were considered as recovery parameters. ${ }^{[10]}$

\section{Statistical Analysis of Data}

All the data were expressed as mean \pm SD. Normal VF group and high VF group were compared and analyzed using independent Student's $t$ test. Independent association of SBP and DBP with TBF and VFL was assessed by regression analysis. $P$ value $<0.05$ was considered as statistically significant.

\section{RESULTS}

There was no significant difference in age, height and physical activity between normal and high VF groups in both genders. There was significant increase $(\mathrm{p}<0.001)$ in body weight, BMI, WC, TBF, FM, FFM in both male and female high VF groups compared to normal VF groups. Cardiovascular parameters like resting SBP, DBP were significantly increased in high VF groups of both genders. Though RPP was increased in both genders of high VF group, it was statistically significant only in female group $(p<0.001)$. There was no significant difference in resting heart rate between two groups of both genders. During various stages of exercise, heart rate was increased in both groups but more in high VF group as compared to normal VF groups. Abnormal heart rate responses were not seen in any groups (Table 1).

HR at various stages of exercise in male adolescents was shown in Table 2. There was no significant difference in baseline HR between the groups. From stage 1 to stage 4, HR was higher in high VF group than normal VF group and it was statistically significant. The baseline SBP was higher in high VF group than normal group of male adolescents, and was statistically significant too between the groups. From stage 2 to stage 4 , SBP was significantly increased in high VF group than normal VF group (Table 3). The baseline DBP was higher in high VF group than normal group and it was statistically significant. There was significant increase in DBP from stage 1 to stage 3 and at $4^{\text {th }}$ and $6^{\text {th }}$ min of recovery in high VF group compared to normal VF (Table 4). There was no difference in the baseline RPP between the groups. From stage 1 to 4 , RPP was significantly higher in high VF group than normal VF group (Table 5).

For a unit rise in TBF percentage, there was an expected increase of $0.294(\mathrm{mmHg})$ units in SBP which was statistically not significant in male adolescents. For a unit rise in VFL, there was an expected increase of 0.312 ( $\mathrm{mmHg}$ ) units in SBP which was statistically not significant in male adolescents. Adjusted $\mathrm{R}^{2}=0.109$, means that the model (VFL and TBF variables) explains $10.90 \%$ observed variations in SBP 2 in male adolescents (Table 6).

For a unit rise in TBF percentage, there was an expected increase of $0.002(\mathrm{mmHg})$ units in DBP which was statistically not significant in male adolescents. For a unit rise in VFL, there was an expected increase of 0.423 ( $\mathrm{mmHg}$ ) units in DBP which was statistically not significant in male adolescents. Adjusted $\mathrm{R}^{2}=0.084$ means that the model (VFL and TBF variables) explains $8.4 \%$ observed variations in DBP 2 in male adolescents (Table 7)

There was no significant difference in the baseline HR between two groups of female adolescents. HR was significantly increased from stage 1 to stage 3 and in female high VF group compared to normal VF group (Table 8).

SBP was higher in high VF group of female adolescents, during resting, stage 1 to stage 4 and even during recovery period at $2^{\text {nd }}, 4^{\text {th }}$ and $6^{\text {th }}$ min 
Table 1: Basic characteristics of participants in relation to visceral fat level.

\begin{tabular}{|c|c|c|c|c|}
\hline \multirow[b]{2}{*}{ Variables } & \multicolumn{2}{|c|}{ Male } & \multicolumn{2}{|c|}{ Female } \\
\hline & $\begin{array}{l}\text { Normal VF Group } \\
\qquad(n=30)\end{array}$ & $\begin{array}{l}\text { High VF Group } \\
\qquad(n=30)\end{array}$ & $\begin{array}{l}\text { Normal VF Group } \\
\qquad(n=30)\end{array}$ & $\begin{array}{l}\text { High VF Group } \\
\qquad(n=30)\end{array}$ \\
\hline Age (yr) & $18.1 \pm 0.30$ & $18.23 \pm 0.43$ & $18.53 \pm 0.50$ & $18.60 \pm 0.49$ \\
\hline PA-R & $1.93 \pm 0.90$ & $1.53 \pm 0.81$ & $0.96 \pm 0.61$ & $1 \pm 0.64$ \\
\hline Height (m) & $1.70 \pm 0.062$ & $1.71 \pm 0.051$ & $1.56 \pm 0.065$ & $1.56 \pm 0.070$ \\
\hline Weight (kg) & $60.10 \pm 8.15$ & $87.51 \pm 11.99^{* *}$ & $55.01 \pm 7.43$ & $84.90 \pm 11.50^{\star *}$ \\
\hline BMI $\left(\mathrm{kg} / \mathrm{m}^{2}\right)$ & $20.73 \pm 2.31$ & $29.86 \pm 3.63^{* *}$ & $22.74 \pm 3.51$ & $34.37 \pm 2.95^{\star \star}$ \\
\hline $\mathrm{WC}(\mathrm{cm})$ & $75.8 \pm 6.84$ & $102.81 \pm 9.31^{\star *}$ & $77.55 \pm 7.59$ & $100.30 \pm 7.15^{\star *}$ \\
\hline TBF\% & $18.02 \pm 5.24$ & $28.63 \pm 3.78^{\star *}$ & $30.36 \pm 4.71$ & $39.69 \pm 2.01^{* *}$ \\
\hline $\mathrm{FM}(\mathrm{kg})$ & $11.07 \pm 4.42$ & $25.41 \pm 6.85^{\star *}$ & $16.94 \pm 4.48$ & $33.83 \pm 5.94^{* *}$ \\
\hline FFM & $49.02 \pm 5.17$ & $62.09 \pm 5.79^{* *}$ & $38.06 \pm 3.84$ & $51.06 \pm 5.92^{\star *}$ \\
\hline $\mathrm{HR}$ & $87.1 \pm 10.44$ & $86.88 \pm 8.01$ & $89.46 \pm 6.80$ & $92.16 \pm 10.67$ \\
\hline SBP & $117 \pm 6.53$ & $120.65 \pm 6.54^{*}$ & $104.06 \pm 7.73$ & $115.06 \pm 6.74^{\star *}$ \\
\hline DBP & $69.76 \pm 4.81$ & $74.58 \pm 6.15^{\star *}$ & $67.66 \pm 7.68$ & $75.73 \pm 6.43^{\star}$ \\
\hline RPP & $102.31 \pm 15.21$ & $104.85 \pm 11.37$ & $93.29 \pm 11.47$ & $106.11 \pm 14.65^{\star *}$ \\
\hline
\end{tabular}

The data were expressed as mean \pm SD. P value $<0.05$ was considered statistically significant. PA: Physical activity; BMI: Body mass index; WC: Waist circumference; TBF: Total body fat; FM: Fat mass; FFM: Fat free mass; HR: Heart rate; SBP: Systolic blood pressure; DBP: Diastolic blood pressure; RPP: Rate pressure product ${ }^{* *} \mathrm{p}<0.001 ;{ }^{*} \mathrm{p}<0.05$

Table 2: Heart Rate at various stages of exercise in male adolescents.

\begin{tabular}{cccccc}
$\begin{array}{c}\text { HR } \\
(\mathbf{b p m})\end{array}$ & $\begin{array}{c}\text { Normal VF group } \\
(\mathbf{n}=\mathbf{3 0})\end{array}$ & $\begin{array}{c}\text { High VF Group } \\
(\mathbf{n}=\mathbf{3 0})\end{array}$ & $\mathbf{P}$ \\
Basal or Resting & $87.1 \pm 10.44$ & 30 & $86.88 \pm 8.01$ & 30 & 0.929 \\
Stage 1 & $114.16 \pm 11.63$ & 30 & $120.86 \pm 12.85$ & 30 & 0.038 \\
Stage 2 & $121.5 \pm 12.07$ & 30 & $129.31 \pm 9.86$ & 29 & 0.008 \\
Stage 3 & $134.4 \pm 12.40$ & 30 & $149.34 \pm 13.47$ & 29 & $<0.001$ \\
Stage 4 & $151.62 \pm 13.07$ & 29 & $168.78 \pm 4.80$ & 23 & $<0.001$ \\
Stage 5 & $168.60 \pm 5.92$ & 23 & $171.66 \pm 0.57$ & 3 & 0.388 \\
$\begin{array}{c}\text { Stage 6 } \\
\text { Recovery at 2 } \\
\text { min }\end{array}$ & $1171.25 \pm 2.22$ & 4 & NA & 0 & NA \\
$\begin{array}{c}\text { Recovery at 4 } \\
\text { min }\end{array}$ & $104.63 \pm 9.63$ & 30 & $100.66 \pm 8.82$ & 30 & 0.101 \\
$\begin{array}{c}\text { Recovery at 6 } \\
\text { min }\end{array}$ & $100.1 \pm 9.57$ & 30 & $97.56 \pm 9.40$ & 30 & 0.305 \\
\hline
\end{tabular}

The data were expressed as mean \pm SD. $P$ value $<0.05$ was considered statistically significant. HR: Heart rate; bpm: Beats per minute

and was statistically significant between normal VF and high VF groups (Table 9).

DBP was significantly increased in high TBF group of female adolescents during baseline, from stage 1 to stage 3 and recovery period at $2^{\text {nd }}$ and $6^{\text {th }} \min$ (Table 10).

RPP was significantly increased in high VF group of female adolescents during resting, stage 1 to 4 and during recovery period at $2^{\text {nd }}, 4^{\text {th }}$ and $6^{\text {th }}$ min compared to normal VF group (Table 11).
Table 3: Systolic blood pressure at various stages of exercise in male adolescents.

\begin{tabular}{|c|c|c|c|c|c|}
\hline \multirow{2}{*}{$\begin{array}{l}\text { SBP }(\mathbf{m m H g}) \\
\text { Basal or Resting }\end{array}$} & \multicolumn{2}{|c|}{$\begin{array}{c}\text { Normal VF Group } \\
(n=30)\end{array}$} & \multicolumn{2}{|c|}{$\begin{array}{l}\text { High VF Group } \\
\qquad(\mathrm{n}=30)\end{array}$} & \multirow{2}{*}{$\begin{array}{c}\mathbf{P} \\
0.045\end{array}$} \\
\hline & $117 \pm 6.53$ & 30 & $120.65 \pm 6.54$ & 30 & \\
\hline Stage 1 & $132.8 \pm 8.52$ & 30 & $136.1 \pm 12.86$ & 30 & 0.246 \\
\hline Stage 2 & $134.36 \pm 10.81$ & 30 & $140.58 \pm 11.06$ & 29 & 0.033 \\
\hline Stage 3 & $140.16 \pm 8.14$ & 30 & $148.00 \pm 13.01$ & 29 & 0.007 \\
\hline Stage 4 & $142.62 \pm 14.21$ & 29 & $152.91 \pm 18.99$ & 23 & 0.029 \\
\hline Stage 5 & $145.78 \pm 12.38$ & 23 & $139.33 \pm 18.92$ & 3 & 0.428 \\
\hline Stage 6 & $142.75 \pm 7.85$ & 4 & NA & 0 & NA \\
\hline $\begin{array}{c}\text { Recovery at } 2 \\
\text { min }\end{array}$ & $134.13 \pm 9.11$ & 30 & $137.2 \pm 9.73$ & 30 & 0.212 \\
\hline $\begin{array}{c}\text { Recovery at } 4 \\
\text { min }\end{array}$ & $126.56 \pm 7.50$ & 30 & $130.43 \pm 9.57$ & 30 & 0.086 \\
\hline $\begin{array}{c}\text { Recovery at } 6 \\
\text { min }\end{array}$ & $122.53 \pm 7.14$ & 30 & $125.43 \pm 8.28$ & 30 & 0.152 \\
\hline
\end{tabular}

The data were expressed as mean \pm SD. P value $<0.05$ was considered statistically significant. SBP: Systolic blood pressure

For a unit rise in TBF percentage, there was an expected fall of 0.268 ( $\mathrm{mmHg}$ ) units in SBP which was statistically not significant with 95\% CI $(-1.440,0.903)$ in female adolescents. While, for a unit rise in VFL there was an expected increase of $1.179(\mathrm{mmHg})$ units in SBP which was statistically significant with $95 \%$ CI $(0.060,2.29)$ in female adolescents. Adjusted $\mathrm{R}^{2}=0.172$ means that the model (VFL and TBF variables) explains $17.20 \%$ observed variations in SBP 2 in female adolescents (Table 12). 
Table 4: Diastolic blood pressure at various stages of exercise in male aadolescents.

\begin{tabular}{cccccc}
$\begin{array}{c}\text { DBP } \\
(\mathbf{m m H g})\end{array}$ & $\begin{array}{c}\text { Normal VF Group } \\
(\mathbf{n = 3 0})\end{array}$ & $\begin{array}{c}\text { High VF Group } \\
(\mathbf{n = 3 0 )}\end{array}$ & $\mathbf{P}$ \\
\hline Basal or Resting & $69.76 \pm 4.81$ & 30 & $74.58 \pm 6.15$ & 30 & $<0.001$ \\
Stage 1 & $77.96 \pm 5.97$ & 30 & $83.03 \pm 9.14$ & 30 & 0.013 \\
Stage 2 & $78.1 \pm 5.87$ & 30 & $82.72 \pm 7.48$ & 29 & 0.010 \\
Stage 3 & $78.53 \pm 6.02$ & 30 & $84.27 \pm 7.93$ & 29 & 0.002 \\
Stage 4 & $78.51 \pm 9.78$ & 29 & $82.73 \pm 11.57$ & 23 & 0.160 \\
$\begin{array}{c}\text { Stage 5 } \\
\text { Stage 6 }\end{array}$ & $80.39 \pm 9.08$ & 23 & $77.66 \pm 14.01$ & 3 & 0.647 \\
$\begin{array}{c}\text { Recovery at 2 } \\
\text { min }\end{array}$ & $75 \pm 15.03$ & 4 & $\mathrm{NA}$ & 0 & $\mathrm{NA}$ \\
$\begin{array}{c}\text { Recovery at 4 } \\
\text { min }\end{array}$ & $74.03 \pm 6.20$ & 30 & $77.66 \pm 7.82$ & 30 & 0.050 \\
$\begin{array}{c}\text { Recovery at 6 } \\
\text { min }\end{array}$ & $73.2 \pm 5.41$ & 30 & $77.23 \pm 6.92$ & 30 & 0.014 \\
\hline
\end{tabular}

The data were expressed as mean \pm SD. P value $<0.05$ was considered statistically significant. DBP: Diastolic blood pressure

Table 5: Rate pressure product at various stages of exercise in male adolescents.

$\begin{array}{cccccc}\begin{array}{c}\text { RPP } \\ \text { (mmHg.bpm) }\end{array} & \text { Normal VF Group } & \text { High VF Group } & \text { P } \\ \text { Basal or Resting } & 102.31 \pm 15.21 & 30 & 104.85 \pm 11.37 & 30 & 0.467 \\ \text { Stage 1 } & 151.67 \pm 18.73 & 30 & 165.11 \pm 29.13 & 30 & 0.037 \\ \text { Stage 2 } & 163.21 \pm 20.67 & 30 & 179.22 \pm 22.57 & 29 & 0.006 \\ \text { Stage 3 } & 188.32 \pm 20.03 & 30 & 221.05 \pm 27.81 & 29 & <0.001 \\ \text { Stage 4 } & 216.50 \pm 30.29 & 29 & 258.14 \pm 33.23 & 23 & <0.001 \\ \text { Stage 5 } & 245.57 \pm 20.08 & 23 & 239.23 \pm 33 & 3 & 0.634 \\ \text { Stage 6 } & 244.57 \pm 16.22 & 4 & \mathrm{NA} & 0 & \mathrm{NA} \\ \text { Recovery at 2 min } & 150.93 \pm 19.13 & 30 & 147.70 \pm 20.24 & 30 & 0.527 \\ \text { Recovery at 4 min } & 132.58 \pm 15.84 & 30 & 131.43 \pm 15.96 & 30 & 0.781 \\ \text { Recovery at 6 min } & 122.91 \pm 16.10 & 30 & 122.51 \pm 15.61 & 30 & 0.924\end{array}$

The data were expressed as mean $\pm S D$. $P$ value $<0.05$ was considered statistically significant. RPP: Rate pressure product

Table 6: Regression analysis of SBP 2 (SBP during stage 2 of Bruce Treadmill Test) for male adolescents.

\begin{tabular}{|c|c|c|c|c|c|}
\hline \multirow{2}{*}{$\begin{array}{c}\text { Independent } \\
\text { Variables }\end{array}$} & \multirow{2}{*}{$\begin{array}{c}\text { Standardized } \\
\text { Regression } \\
\text { coefficient } \beta\end{array}$} & \multicolumn{2}{|c|}{$\begin{array}{l}\text { Confidence } \\
\text { interval }\end{array}$} & \multirow{2}{*}{$P$} & \multirow{2}{*}{$\mathbf{R}^{2}$} \\
\hline & & $\begin{array}{l}\text { Upper } \\
\text { limit }\end{array}$ & $\begin{array}{l}\text { Lower } \\
\text { limit }\end{array}$ & & \\
\hline TBF & 0.294 & -0.549 & 1.13 & 0.487 & \multirow{2}{*}{0.109} \\
\hline VFL & 0.312 & -0.745 & 1.37 & 0.556 & \\
\hline
\end{tabular}

$P$ value $<0.05$ was considered statistically significant. SBP: Systolic blood pressure; TBF: Total body fat; VFL: Visceral fat level
Table 7: Regression analysis of DBP 2 (DBP during stage 2 of Bruce Treadmill Test) for male adolescents.

\begin{tabular}{|c|c|c|c|c|c|}
\hline \multirow{2}{*}{$\begin{array}{c}\text { Independent } \\
\text { Variables }\end{array}$} & \multirow{2}{*}{$\begin{array}{l}\text { Standardized } \\
\beta \text { Co-efficient }\end{array}$} & \multicolumn{2}{|c|}{ Confidence interval } & \multirow{2}{*}{$\mathbf{P}$} & \multirow{2}{*}{$\mathbf{R}^{2}$} \\
\hline & & $\begin{array}{c}\text { Upper } \\
\text { Limit }\end{array}$ & $\begin{array}{l}\text { Lower } \\
\text { limit }\end{array}$ & & \\
\hline TBF & 0.002 & -0.523 & 0.527 & 0.994 & \\
\hline VFL & 0.423 & -0.234 & 1.08 & 0.203 &. \\
\hline
\end{tabular}

$P$ value $<0.05$ was considered statistically significant. DBP: Diastolic blood pressure; TBF: Total body fat; VFL: Visceral fat level

Table 8: Heart rate at various stages of exercise in female adolescents.

\begin{tabular}{cccccc}
$\begin{array}{c}\text { HR } \\
\text { (bpm) }\end{array}$ & \multicolumn{2}{c}{ Normal VF Group } & High VF Group & P \\
$\begin{array}{c}\text { Basal or } \\
\text { Resting }\end{array}$ & $89.46 \pm 6.80$ & 30 & $92.16 \pm 10.67$ & 30 & 0.247 \\
Stage 1 & $124 \pm 9.91$ & 30 & $134.43 \pm 13.74$ & 30 & 0.001 \\
Stage 2 & $133.36 \pm 9.12$ & 30 & $147.65 \pm 13.73$ & 29 & $<0.001$ \\
Stage 3 & $150.66 \pm 10.12$ & 30 & $164.91 \pm 8.77$ & 23 & $<0.001$ \\
Stage 4 & $167.42 \pm 6.15$ & 28 & $170.42 \pm 0.97$ & 7 & 0.212 \\
$\begin{array}{c}\text { Stage 5 } \\
\text { Stage 6 }\end{array}$ & $170.67 \pm 1.03$ & 6 & $\mathrm{NA}$ & 0 & $\mathrm{NA}$ \\
$\begin{array}{c}\text { Recovery at } \\
2 \text { min }\end{array}$ & $111.26 \pm 11.36$ & 30 & $109.33 \pm 10.82$ & 30 & 0.502 \\
$\begin{array}{c}\text { Recovery at } \\
4 \text { min }\end{array}$ & $104.76 \pm 10.38$ & 30 & $103.5 \pm 9.72$ & 30 & 0.627 \\
$\begin{array}{c}\text { Recovery at } \\
6 \text { min }\end{array}$ & $101.6 \pm 9.36$ & 30 & $101.86 \pm 9.81$ & 30 & 0.914 \\
\hline
\end{tabular}

The data were expressed as mean \pm SD. P value $<0.05$ was considered statistically significant. HR: Heart rate; bpm: Beats per minute

Table 9: Systolic blood pressure at various stages of exercise in female adolescents.

$\begin{array}{cccccc}\begin{array}{c}\text { SBP } \\ (\mathrm{mmHg})\end{array} & \text { Normal VF Group } & \text { High VF Group } & \text { P } \\ \begin{array}{c}\text { Basal or } \\ \text { Resting }\end{array} & 104.06 \pm 7.73 & 30 & 115.06 \pm 6.74 & 30 & <0.001 \\ \text { Stage 1 } & 119.13 \pm 8.52 & 30 & 133.63 \pm 13.91 & 30 & <0.001 \\ \text { Stage 2 } & 121.96 \pm 10.10 & 30 & 135.93 \pm 14.75 & 29 & <0.001 \\ \text { Stage 3 } & 124.46 \pm 11.16 & 30 & 142.95 \pm 10.42 & 23 & <0.001 \\ \text { Stage } 4 & 126.5 \pm 10.36 & 28 & 148.71 \pm 11.62 & 7 & <0.001 \\ \text { Stage 5 } & 119 \pm 14.99 & 6 & \mathrm{NA} & 0 & \mathrm{NA} \\ \begin{array}{c}\text { Stage 6 } \\ \text { Recovery at } \\ 2 \text { min }\end{array} & 115.33 \pm 6.92 & 30 & 125.9 \pm 9.25 & 30 & <0.001 \\ \text { Recovery at } & 109.96 \pm 6.88 & 30 & 121 \pm 8.58 & 30 & <0.001 \\ 4 \text { min } & & & & & \\ \text { Recovery at } & 104.86 \pm 19.09 & 30 & 117.53 \pm 8.77 & 30 & 0.001 \\ 6 \text { min } & & & & & \end{array}$

The data were expressed as mean \pm SD. P value $<0.05$ was considered statistically significant. SBP: Sysstolic blood pressure 
Table 10: Diastolic blood pressure at various stages of exercise in female adolescents.

\begin{tabular}{|cccccc} 
DBP $(\mathrm{mmHg})$ & Normal TBF Group & \multicolumn{2}{c}{ High TBF Group } & P \\
Basal or Resting & $65.46 \pm 6.15$ & 13 & $73.42 \pm 7.78$ & 47 & 0.001 \\
Stage 1 & $74 \pm 7.21$ & 13 & $80.97 \pm 10.11$ & 47 & 0.023 \\
Stage 2 & $74.30 \pm 8.34$ & 13 & $81.65 \pm 9.36$ & 46 & 0.013 \\
Stage 3 & $74.23 \pm 7.94$ & 13 & $81.25 \pm 11.01$ & 40 & 0.039 \\
Stage 4 & $72.76 \pm 9.63$ & 13 & $81.81 \pm 16.36$ & 22 & 0.079 \\
Stage 5 & $78 \pm 13.85$ & 3 & $72.66 \pm 10.96$ & 3 & 0.628 \\
Stage 6 & & & $\mathrm{NA}$ & & \\
Recovery at 2 min & $67.84 \pm 7.55$ & 13 & $73.85 \pm 8.22$ & 47 & 0.021 \\
Recovery at 4 min & $69.38 \pm 7.56$ & 13 & $74.21 \pm 8.34$ & 47 & 0.064 \\
Recovery at 6 min & $69.38 \pm 7.78$ & 13 & $74.65 \pm 8.39$ & 47 & 0.046
\end{tabular}

The data were expressed as mean \pm SD. P value $<0.05$ was considered statistically significant. DBP: Diastolic blood pressure

Table 11: Rate pressure product at various stages of exercise in female adolescents.

\begin{tabular}{cccccc}
$\begin{array}{c}\text { RPP }(\mathrm{mmHg} . \\
\text { bp) }\end{array}$ & \multicolumn{2}{c}{ Normal VF Group } & High VF Group & P \\
$\begin{array}{c}\text { Basal or } \\
\text { Resting }\end{array}$ & $93.29 \pm 11.47$ & 30 & $106.11 \pm 14.65$ & 30 & $<0.001$ \\
Stage 1 & $148.09 \pm 18.94$ & 30 & $180.35 \pm 31.18$ & 30 & $<0.001$ \\
Stage 2 & $162.75 \pm 18.18$ & 30 & $201.11 \pm 32.75$ & 29 & $<0.001$ \\
Stage 3 & $187.62 \pm 21.54$ & 30 & $235.60 \pm 19.82$ & 23 & $<0.001$ \\
Stage 4 & $211.88 \pm 19.99$ & 28 & $253.42 \pm 19.36$ & 7 & $<0.001$ \\
Stage 5 & $203.12 \pm 25.75$ & 6 & NA & 0 & NA \\
$\begin{array}{c}\text { Stage 6 } \\
\text { Recovery at 2 } \\
\text { min }\end{array}$ & $128.43 \pm 16.04$ & 30 & $137.96 \pm 19.86$ & 30 & 0.045 \\
$\begin{array}{c}\text { Recovery at 4 } \\
\text { min }\end{array}$ & $115.22 \pm 13.81$ & 30 & $125.39 \pm 16.38$ & 30 & 0.011 \\
$\begin{array}{c}\text { Recovery at 6 } \\
\text { min }\end{array}$ & $106.96 \pm 23.26$ & 30 & $119.86 \pm 15.89$ & 30 & 0.015
\end{tabular}

The data were expressed as mean \pm SD. P value $<0.05$ was considered statistically significant. RPP: Rate pressure product

For a unit rise in TBF\%, there was an expected rise of $0.235(\mathrm{mmHg})$ units in DBP which was statistically not significant with 95\% CI (-0.504, 0.975) in female adolescents. While, for a unit rise in VFL, there was increase of $0.596(\mathrm{mmHg})$ units in DBP, which was statistically significant with 95\% CI $(-0.109,1.302)$ in female adolescents. Adjusted $\mathrm{R}^{2}=0.261$ means that the model (VFL and TBF variables) explains $26.15 \%$ observed variations in DBP 2 in female adolescents (Table 13).

\section{DISCUSSION}

The main objective of the present study was to assess cardiovascular responses to treadmill exercise stress in Indian adolescents with normal
Table 12: Regression analysis of SBP 2 (SBP during stage 2 of Bruce Treadmill Test) for female adolescents.

\begin{tabular}{|c|c|c|c|c|c|}
\hline \multirow{2}{*}{$\begin{array}{c}\text { Independent } \\
\text { Variables }\end{array}$} & \multirow{2}{*}{$\begin{array}{l}\text { Standardized } \\
\text { regression } \\
\text { coefficient } \beta\end{array}$} & \multicolumn{2}{|c|}{$\begin{array}{l}\text { Confidence } \\
\text { interval }\end{array}$} & \multirow[b]{2}{*}{$\mathbf{P}$} & \multirow[b]{2}{*}{$\mathbf{R}^{2}$} \\
\hline & & $\begin{array}{l}\text { Lower } \\
\text { limit }\end{array}$ & $\begin{array}{l}\text { Upper } \\
\text { limit }\end{array}$ & & \\
\hline TBF & -0.268 & -1.440 & 0.903 & 0.649 & \multirow{2}{*}{0.172} \\
\hline VFL & 1.179 & 0.060 & 2.29 & 0.039 & \\
\hline
\end{tabular}

P value $<0.05$ was considered statistically significant. SBP: Systolic blood pressure; TBF: Total body fat; VFL: Visceral fat level

Table 13: Regression Analysis of DBP2 (DBP during stage 2 of Bruce Treadmill Test) for female adolescents.

\begin{tabular}{|c|c|c|c|c|c|}
\hline \multirow{2}{*}{$\begin{array}{l}\text { Independent } \\
\text { Variables }\end{array}$} & \multirow{2}{*}{$\begin{array}{c}\text { Standardized } \\
\text { regression } \\
\text { coefficient } \beta\end{array}$} & \multicolumn{2}{|c|}{$\begin{array}{c}\text { Confidence } \\
\text { interval }\end{array}$} & \multirow[b]{2}{*}{$\mathbf{P}$} & \multirow{2}{*}{$\mathbf{R}^{2}$} \\
\hline & & $\begin{array}{l}\text { Lower } \\
\text { limit }\end{array}$ & $\begin{array}{l}\text { Upper } \\
\text { limit }\end{array}$ & & \\
\hline TBF & 0.235 & -0.504 & 0.975 & 0.527 & \multirow{2}{*}{0.261} \\
\hline VFL & 0.596 & -1.09 & 1.302 & 0.096 & \\
\hline
\end{tabular}

$\mathrm{P}$ value $<0.05$ was considered statistically significant. DBP: Diastolic blood pressure; TBF: Total body fat; VFL: Visceral fat level

visceral fatness and high visceral fatness. The secondary objective of the study was to assess the effect of visceral fatness and total fatness to cardiovascular response to treadmill exercise stress test in Indian adolescents. We found that resting SBP, DBP and RPP (myocardial workload) were increased in high VF group of both male and female adolescents. Stephen R, et al. and Daniel SR, et al. concluded that there is a positive relationship between central fat and hypertension in adolescents and children, ${ }^{[11,12]}$ Hiuge-Shimizu A. et al. stated that raised blood pressure is seen in Japanese with more visceral fat area. ${ }^{[13]}$

In our study, increased SBP, HR and RPP were observed in both groups during various stages of exercise. SBP, DBP and RPP were increased more during exercise in participants with high visceral fat as compared to normal visceral fat. During exercise, myocardial requirement of oxygen increases with intensity of exercise. During successive stage of exercise, increased demand of oxygen or cardiac output are met by sympathetic stimulation in moderately active adolescents. Sympathetic over-activation was seen in form of increased SBP and HR in adolescents with high visceral fat in comparison to normal visceral fat at same exercise load. Reactivity to exercise with same exercise load is different in normotensive adolescents with different body fat mass. Increased reactivity to exercise stress was seen mainly due to high resting blood pressures in high VF group. As such there was no abnormal or exaggerated blood pressure reactivity in high VF group. The findings of our study were supported by Dipla K, et al. ${ }^{[14]}$ Sieira MC, et al. documented that DBP may remain unchanged or somewhat decline during acute dynamic exercise. ${ }^{[15]}$ In our study also, DBP remained same in male adolescents with normal and high VF group. Similarly, increased DBP was seen in female adolescents with high visceral fat during progressive stages of exercise. Miyai N, et al. stated that in normal subjects, a marked increase in DBP is indicative of impaired ability for exercise-induced vasodilatation and elevated basal peripheral resistance. ${ }^{[16]}$ Thus, this theory can be elucidated by an exaggerated activity of the sympathetic nerves, elevated vascular reaction to sympathetic stimulation or by a thickening of arteriolar wall that modifies its ability to react to vasoconstrictor stimuli. In subjects with this type of vascular features and hemodynamic pattern, the increased cardiac output will cause rise in both, SBP and DBP which is 
a characteristic of known case of hypertensive patient. Therefore, both SBP and DBP are key determinant for checking abnormal cardiovascular reactivity to physical stress.

In our study, all normotensive adolescents were free from diabetes and other chronic diseases. High basal blood pressure is considered as one of the independent risk for the development of future hypertension. In our study, resting blood pressure was high in high VF groups and statistically significant as compared to normal VF group. Hence, BP was shooting up more during exercise. Tzemos $\mathrm{N}$, et al. also supported this as he stated that resting BP should be taken into consideration because participants with high exercise BP also tended to demonstrate higher resting cardio vascular parameters. ${ }^{[17]}$ Moreover, Spartano NL stated that elevated basal SBP is commonly related with exaggerated SBP response during exercise. ${ }^{[18]}$

Strength of this study includes BCA and exercise testing was done at the same time for all the participants to maintain homogeneity of the study. Moreover, its concept of analysis visceral fatness and to search blood pressure reactivity in different group of visceral fatness. Visceral fatness can be easily measured by non-invasive bioelectrical impedance method. Normal weight obesity is new emerging problem in Indians. Early detection of hypertension in adolescents helps to make preventive strategy for younger people. Exercise stress is helpful in unmasking future hypertension in adolescents.

\section{Limitations of the Study}

Major limitation of this study is that data collected in a small section of population once and longitudinal study was not planned. Concept of future hypertension emphasized only if data are collected for so many times at short interval of time.

\section{CONCLUSION}

Blood pressure reactivity to treadmill exercise stress test was increased in high visceral fat adolescents as compared to normal visceral fat adolescents, which was primarily due to their high resting blood pressure. Post-exercise recovery was delayed in high visceral fat adolescents compared to normal visceral fat adolescents.

Visceral fat had a stronger influence on baseline cardiovascular parameters like systolic and diastolic blood pressure as well as blood pressure parameter during exercise than total body fat.

\section{ACKNOWLEDGEMENT}

The authors are grateful to the parent organization Charutar Arogya Mandal, Karamsad for providing a platform for the study. The authors are thankful to Dr. Wasim A. Shaikh and Dr. A. R. Nair for their continuous support and guidance. The authors are obliged to our participants without them this study was not possible.

\section{CONFLICT OF INTEREST}

The authors declare that they have no conflict of Interest.

\section{ABBREVIATIONS}

VF: Visceral Fat; BP: Blood Pressure; SBP: Systolic Blood Pressure; BMI: Body Mass Index; BFM: Body Fat Mass; WC: Waist Circumference; TBF: Total Body Fat; FM: Fat Mass; FFM: Fat Free Mass; DBP: Diastolic Blood Pressure.

\section{REFERENCES}

1. Fletcher G, Balady G, Amsterdam E, Chaitman B, Eckel R, Fleg J, et al. Exercise standards for testing and training: A statement for healthcare professionals from the American Heart Association. Circulation. 2001;104(14):1694-740.

2. Kim D, Ha JW. Hypertensive response to exercise: Mechanisms and clinical implication. Clin Hypertens. 2016;22(1):17.

3. Berger A, Grossman E, Katz M, Kivity S, Klempfner R, Segev S, et al. Exercise blood pressure and the risk for future hypertension among normotensive middle-aged adults. J Am Heart Assoc. 2015;4(4):e001710.

4. Esmaeilzadeh S, Ebadollahzadeh K. Physical fitness, physical activity and sedentary activities of 7 to 11 year old boys with different body mass indexes. Asian J Sports Med. 2012;3(2):105-12.

5. Silva LR, Cavaglieri C, Lopes WA, Pizzi J, Coelho-e-Silva MJ, Leite N. Endothelial wall thickness, cardiorespiratory fitness and inflammatory markers in obese and non-obese adolescents. Braz J Phys Ther. 2014;18(1):47-55.

6. Youn JC, Kang SM. Cardiopulmonary exercise test in patients with hypertension: focused on hypertensive response to exercise. Pulse. 2015;3(2):114-7.

7. Lauer MS, Pashkow FJ, Harvey SA, Marwick TH, Thomas JD. Angiographic and prognostic implications of an exaggerated exercise systolic blood pressure response and rest systolic blood pressure in adults undergoing evaluation for suspected coronary artery disease. J Am Coll Cardiol. 1995;26(7):1630-6.

8. Matthews CE, Pate RR, Jackson KL, Ward DS, Macera CA, Kohl HW, et al. Exaggerated blood pressure response to dynamic exercise and risk of future hypertension. J Clin Epidemiol. 1998;51(1):29-35.

9. Stewart KJ, Sung J, Silber HA, Fleg JL, Kelemen MD, et al. Exaggerated exercise blood pressure is related to impaired endothelial vasodilator function. Am J Hypertens. 2004;17(4):314-20.

10. Parikh SM, Shah HD, Singh SK. Does visceral fat affect aerobic fitness in Indian adolescents of 18-19 years' age group?. Natl J Physiol Pharm Pharmacol. 2018;8(2):233-8

11. Daniels SR, Morrison JA, Sprecher DL, Khoury P, KimballTR. Association of body fat distribution and cardiovascular risk factors in children and adolescents. Circulation. 1999;99(4):541-5.

12. Daniels SR, Khoury PR, Morrison JA. Utility of different measures of body fat distribution in children and adolescents. American Journal of Epidemiology. 2000;152(12):1179-84

13. Hiuge-Shimizu A, Kishida K, Funahashi T, Ishizaka Y, Oka R, Okada M, et al. Absolute value of visceral fat area measured on computed tomography scans and obesity-related cardiovascular risk factors in large-scale Japanese general population (the VACATION-J study). Ann Med. 2012;44(1):82-92.

14. Dipla K, Nassis GP, Vrabas IS. Blood pressure control at rest and during exercise in obese children and adults. J Obes. 2012;2012.

15. Sieira MC, Ricart AO, Estrany RS. Blood pressure response to exercise testing. Apunts Medicina de I" Esport. 2010;45(167):191-200.

16. Miyai N, Arita M, Miyashita K, Morioka I, Shiraishi T, Nishio I. Blood pressure response to heart rate during exercise test and risk of future hypertension. Hypertension. 2002;39(3):761-6.

17. Tzemos N, Lim PO, MacDonald TM. Is exercise blood pressure a marker of vascular endothelial function?. QJM. 2002;95(7):423-9.

18. Spartano NL, Lyass A, Larson MG, Lewis GD, Vasan RS. Submaximal exercise systolic blood pressure and heart rate at 20 years of follow-up correlates in the Framingham heart study. J Am Heart Assoc. 2016;5(6):e002821. 